\title{
Chronic itch development in sensory neurons requires BRAF signaling pathways
}

\author{
Zhong-Qiu Zhao,, 1,2 Fu-Quan Huo,, ${ }^{1,2}$ Joseph Jeffry, 1,2 Lori Hampton, ${ }^{3}$ Shadmehr Demehri,4,5 \\ Seungil Kim, ${ }^{1}$ Xian-Yu Liu, ${ }^{1,2}$ Devin M. Barry, ${ }^{1,5}$ Li Wan, ${ }^{1,6}$ Zhong-Chun Liu, ${ }^{1,2}$ Hui Li, ${ }^{1,7}$ Ahu Turkoz, ${ }^{4}$ \\ Kaijie Ma, ${ }^{8}$ Lynn A. Cornelius, ${ }^{1,5}$ Raphael Kopan, ${ }^{4}$ James F. Battey Jr., ${ }^{9}$ \\ Jian Zhong, ${ }^{8,10}$ and Zhou-Feng Chen ${ }^{1,2,4}$

\begin{abstract}
${ }^{1}$ Center for the Study of Itch and 2Departments of Anesthesiology and Psychiatry, Washington University School of Medicine Pain Center, St. Louis, Missouri, USA. ${ }^{3}$ Office of Laboratory Animal Welfare, NIH, Bethesda, Maryland, USA. ${ }^{4}$ Department of Developmental Biology and ${ }^{5}$ Division of Dermatology, Department of Internal Medicine, Washington University School of Medicine, St. Louis, Missouri, USA. ${ }^{6}$ Department of Anesthesiology, The Second Affiliated Hospital, Guangzhou Medical University, Guangzhou, China. ${ }^{7}$ Department of Anatomy, Histology, and Embryology and K.K. Leung Brain Research Centre, The Fourth Military Medical University, Xi'an, China. ${ }^{8}$ Burke Medical Research Institute, Weill Medical College of Cornell University, White Plains, New York, USA. 9National Institute on Deafness and Other Communication Disorders, NIH, Bethesda, Maryland, USA. ${ }^{10}$ Brain and Mind Research Institute, Weill Medical College of Cornell University, New York, New York, USA.
\end{abstract}

\begin{abstract}
Chronic itch, or pruritus, is associated with a wide range of skin abnormalities. The mechanisms responsible for chronic itch induction and persistence remain unclear. We developed a mouse model in which a constitutively active form of the serine/threonine kinase BRAF was expressed in neurons gated by the sodium channel Nav1.8 (BRAF ${ }^{N a v 1.8}$ mice). We found that constitutive BRAF pathway activation in BRAF ${ }^{\text {Nav1.8 }}$ mice results in ectopic and enhanced expression of a cohort of itch-sensing genes, including gastrin-releasing peptide (GRP) and MAS-related GPCR member A3 (MRGPRA3), in nociceptors expressing transient receptor potential vanilloid 1 (TRPV1). BRAF ${ }^{N a v 1.8}$ mice showed de novo neuronal responsiveness to pruritogens, enhanced pruriceptor excitability, and heightened evoked and spontaneous scratching behavior. GRP receptor expression was increased in the spinal cord, indicating augmented coding capacity for itch subsequent to amplified pruriceptive inputs. Enhanced GRP expression and sustained ERK phosphorylation were observed in sensory neurons of mice with allergic contact dermatitis- or dry skin-elicited itch; however, spinal ERK activation was not required for maintaining central sensitization of itch. Inhibition of either BRAF or GRP signaling attenuated itch sensation in chronic itch mouse models. These data uncover RAF/MEK/ERK signaling as a key regulator that confers a subset of nociceptors with pruriceptive properties to initiate and maintain long-lasting itch sensation.
\end{abstract}

\section{Introduction}

The ability of the brain to discriminate pain from itch in order to make binary decisions - eliciting either withdrawal or scratching behavior - is critically dependent on the functional connectivity of the somatosensory system. Itch information, along with pain, is conveyed by primary afferents of the dorsal root ganglion (DRG) to the spinal cord and of the trigeminal ganglion neurons to the trigeminal subnucleus caudalis of the brainstem, respectively, which in turn supplies input to the somatosensory cortex through spinothalamic tract or trigeminothalamic tract neurons (1-4). At the molecular level, emerging evidence suggests that activation of GPCRs in sensory neurons is likely responsible for relaying distinct types of acute stimulus-evoked itch (5-7). In addition, several transient receptor potential (TRP) channels, including TRP vanilloid 1 (TRPV1) and TRPA1, have been implicated in mediating histaminergic and nonhistaminergic itch, respectively $(8-10)$. In the spinal cord, gastrin-releasing peptide (GRP) receptor (GRPR) and neurons expressing GRPR are key mediators dedicated to the coding of itch sensation (11-13). In contrast to acute itch, chronic itch may arise from an altered or diseased state of the

Authorship note: Zhong-Qiu Zhao and Fu-Quan Huo contributed equally to this work.

Conflict of interest: The authors have declared that no conflict of interest exists. Citation for this article: JClin Invest. 2013;123(11):4769-4780. doi:10.1172/JCI70528. skin or immune or nervous systems or from a dysregulation of metabolism. Chronic itch represents a significant clinical problem, largely due to its resistance to most commonly used antihistamine-based therapies $(14,15)$. To date, little is known about signaling mechanisms underlying chronic itch in the nervous system. At the cellular level, mice lacking the transcription factor $B h l b b 5$ in a subset of spinal cord cells showed a heightened itch transmission, presumably resulting from a loss of inhibitory interneurons expressing Bhlhb5 during development (16). In contrast, mice lacking spinal GRPR ${ }^{+}$neurons lack the ability to sense chronic itch (13). In sensory neurons, the conditional deletion of vesicular glutamate transporter 2 (Vglut2) results in spontaneous itch sensation $(17,18)$, and TRPA1 is important for dry skin itch (19). Ablation of neurons expressing MAS-related GPCR member A3 (MRGPRA) attenuates dry skin and allergic itch, suggesting that MRGPRA $3^{+}$neurons are also important for the development of chronic itch (20). Although GRPR has emerged as an important receptor for mediating acute itch, the function of GRPR and its cognate ligand GRP in chronic itch has not been characterized.

$\mathrm{BRAF}$ is a serine/threonine kinase that activates ERK, a member of the MAPKs superfamily, through the RAF/MEK/ERK cascade. Upon activation, pERK triggers the expression of a plethora of transcription factors in the nucleus to regulate a wide array of cellular functions $(21,22)$. Our previous studies of conditional BRAF loss-of-function mice have uncovered an 
A

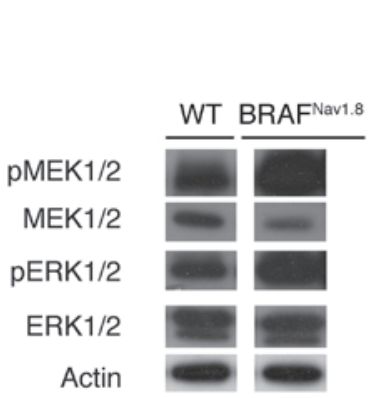

G

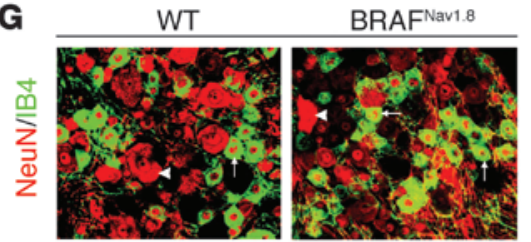

J

J
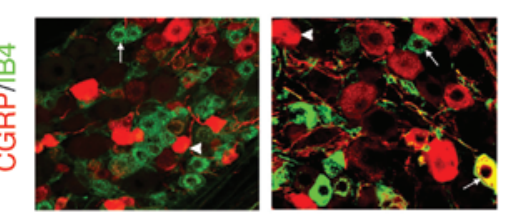

M

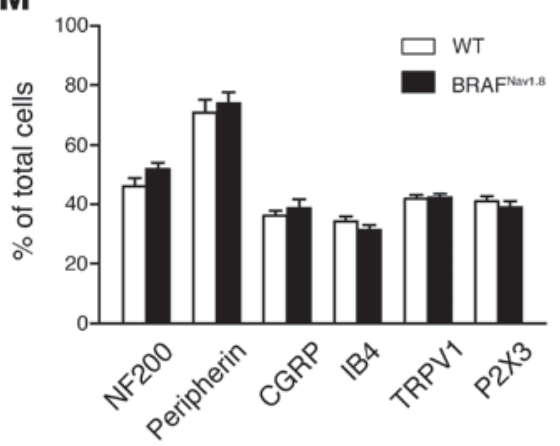

B

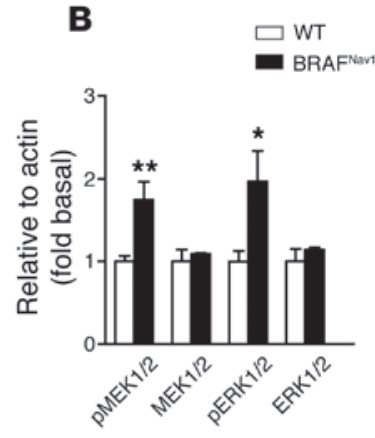

H

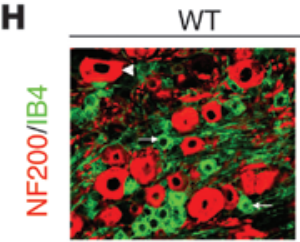

K
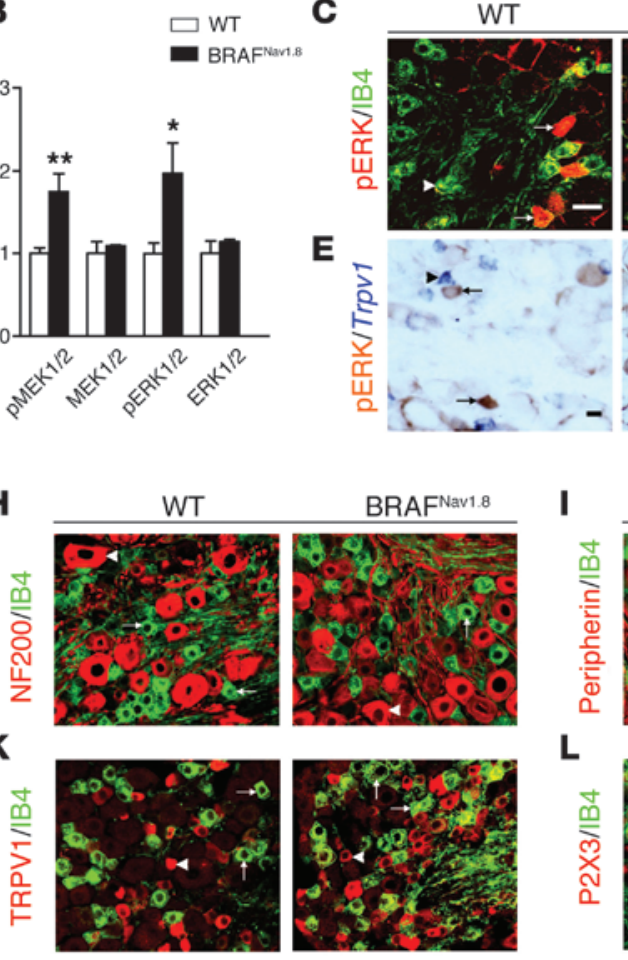

E
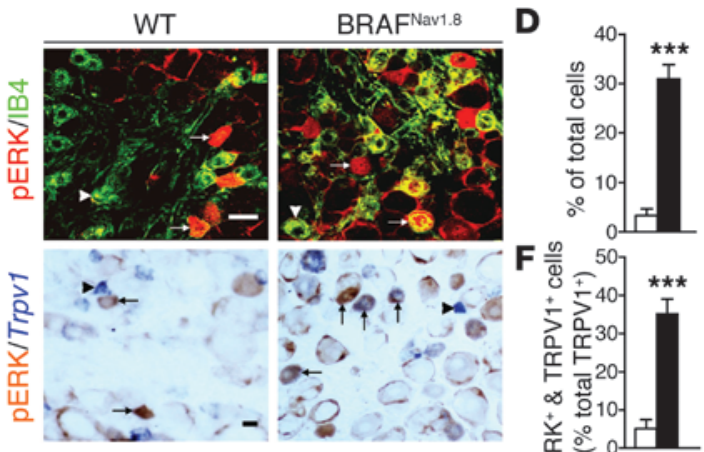

F $\frac{\underline{0}}{\bar{\alpha}}$
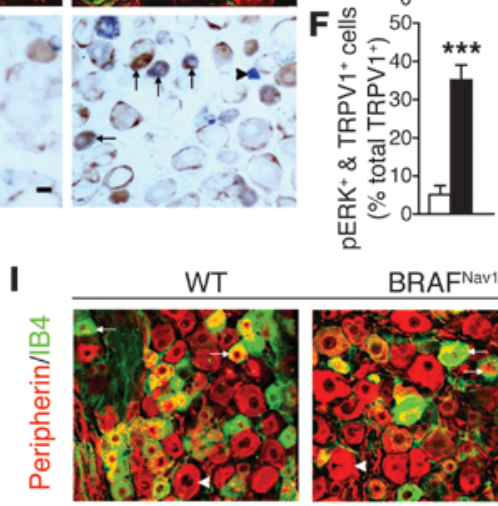

L
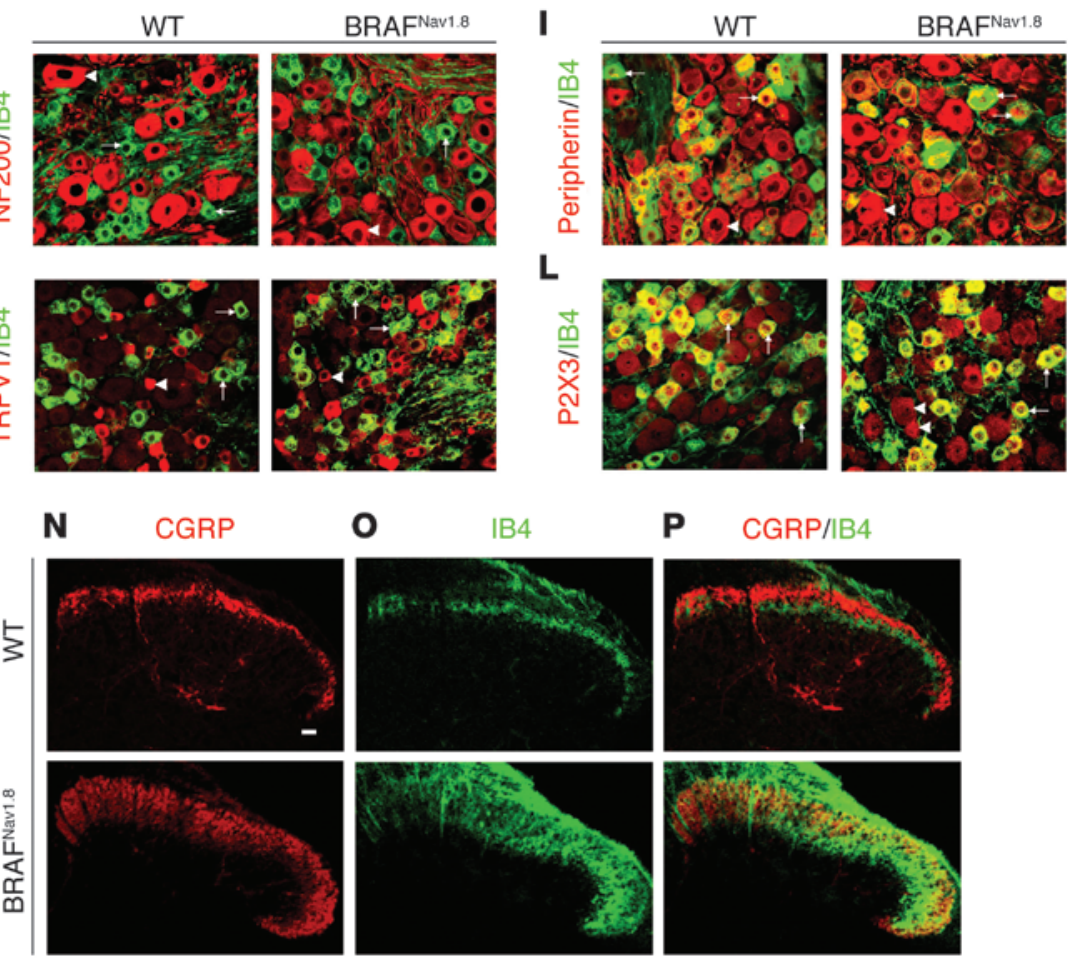

Figure 1

Increased levels of pMEK1/2 and pERK1/2 in sensory neurons and aberrant innervations in the spinal cords of BRAFNav1.8 mice. (A) A representative Western blot and (B) quantified data show increased levels of pMEK1/2 and pERK1/2 but unchanged total MEK $1 / 2$ and ERK1/2 levels in DRGs of BRAFNav1.8 mice. $n=6$ per genotype. All the lanes were run on the same gel but are noncontiguous. ${ }^{*} P<0.05,{ }^{* *} P<0.01$, BRAFNav1.8 mice vs. wild-type. (C-F) Increased pERK ${ }^{+}$cells are indicated by double staining with pERK (red) and IB4 (green) or with pERK (brown) in $\operatorname{Trpv1}^{+}$(blue) cells in BRAFNav1.8 mice compared to wild-type mice. $n=4$ per genotype. ${ }^{* \star *} P<0.001$. (G-L) Expression of molecular markers with IB4 (green) in DRGs of wild-type and BRAFNav1.8 mice. (M) Quantitative comparison of DRG neurons between 2 groups. $n=3$ per genotype. $(\mathbf{N}-\mathbf{P})$ Expression of (N) CGRP and (O) IB4 in the spinal cords of wild-type and BRAFNav1.8 mice. Arrows and arrowheads indicate (C and E) pERK ${ }^{+}$or pERK- cells, respectively, and (G-L) IB4+ or IB4- cells, respectively. Scale bar: $20 \mu \mathrm{m}$.

essential role for RAF signaling in the extension of peripheral sensory projections as well as in the initial development of some of the DRG's molecular makeup and central projections (23). To further explore potential roles of BRAF signaling in sensory transduction, we generated BRAF ${ }^{N a v 1.8}$ mice in which the BRAF kinase is selectively activated in sensory neurons expressing the sodium channel Nav1.8 by genetically replacing the wild-type Braf gene with a kinase-activated one in these neurons $(24,25)$. BRAF $^{\text {Nav1. } 1.8}$ mice exhibited spontaneous scratching behavior, prompting us to hypothesize that BRAF signaling plays a role in pruriceptive transmission.

\section{Results}

Peptidergic and nonpeptidergic fibers maintain their cellular identity but show aberrant central projections in BRAFNav1.8 mice. The mutant ${ }^{V} 600 \mathrm{E} B R A F$ protein was strongly expressed in DRG neurons of BRAFNav1.8 mice (Supplemental Figure 1; supplemental material available online with this article; doi:10.1172/JCI70528DS1), and, as expected, the levels of phosphorylated MEK1/2 and ERK1/2 (pMEK1/2 and pERK1/2) were significantly elevated in DRG neurons of BRAF ${ }^{\text {Nav1. }} .8$ mice relative to those of wild-type mice (Figure 1, $A$ and B). Total BRAF and CRAF expression levels did not differ in DRG neurons between BRAF ${ }^{\text {Nav1.8 }}$ and wild-type mice 

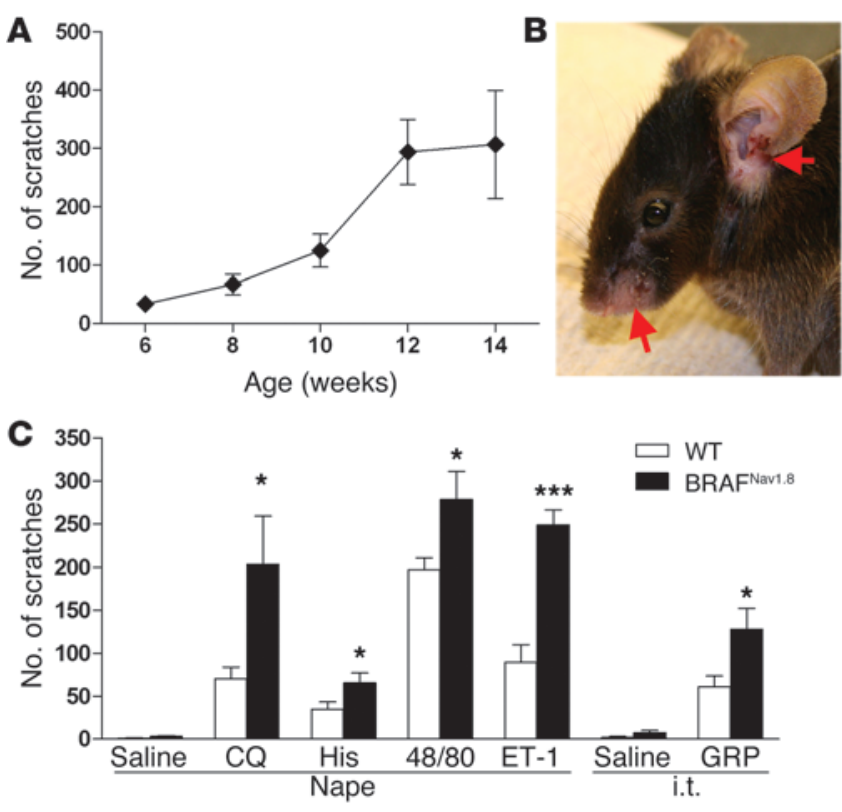

(Supplemental Figure 1). In wild-type mice, $\mathrm{pERK}^{+}$neurons were sparse in DRGs (3.2\%), but they were widespread in DRG neurons of BRAF ${ }^{\mathrm{Nav}} 1.8$ mice $(30.9 \%)$ (Figure $1, \mathrm{C}$ and $\mathrm{D}$ ). The number of $\mathrm{pERK}^{+}$cells increased similarly in DRG neurons at cervical, thoracic, and lumbar segmental levels (Supplemental Figure 2). The density of primary afferent fibers innervating in cervical and lumbar back hairy skin regions also increased in $\mathrm{BRAF}^{\mathrm{Nav} 1.8}$ mice (Supplemental Figure 3). Ectopic pERK was detected in neurons expressing lectin-binding IB4 (a nonpeptidergic marker) and TRPV1, both commonly used markers for subsets of nociceptors (26). The proportion of TRPV1+ neurons expressing PERK increased from $5.0 \%$ to $35.1 \%$ (Figure 1, E and F). In contrast, the proportions of neurons expressing NeuN (neuronal-specific nuclear protein, a neuronal marker), NF200 (a large-diameter and myelinated neuronal marker), peripherin (a small-diameter neuronal marker), CGRP, IB4, TRPV1, and P2X3 (a pain-sensing ATP-gated ion channel also expressed in IB4 ${ }^{+}$cells) were comparable between the 2 groups (Figure 1, G-M). Thus, the fates of peptidergic, nonpeptidergic, and large-diameter neurons were maintained in BRAFNav1.8 mice.

Peptidergic and nonpeptidergic fibers innervate laminae I and II outer layer (IIo) and lamina II inner layer (IIi) in the spinal cord, respectively (Figure 1, N-P). In $\mathrm{BRAF}^{\mathrm{Nav} 1.8}$ mice, $\mathrm{CGRP}^{+}$fibers expanded to lamina IIi and were colocalized with IB4, whereas the nonpeptidergic IB4 $4^{+}$fibers invaded laminae I and IIo domains (Figure 1, N-P). These findings reveal that an appropriate level of BRAF signaling is important in directing the segregation of peptidergic and nonpeptidergic primary afferents to their respective target zones of the spinal cord.

Spontaneous and enhanced evoked scratching behavior of BRAF $F^{\text {Nav1.8 }}$ mice. At around 6 weeks of age, about $50 \%$ of BRAFNav1.8 mice showed spontaneous scratching behavior, followed by skin lesions (Figure 2, A and B, and Supplemental Video 1). By 2 months of age, the majority of BRAF ${ }^{N a v 1.8}$ mice had visible skin lesions, most notably around the craniofacial areas (Figure $2 \mathrm{~B}$ ) and the neck region. Prior to the onset of spontaneous scratching, BRAFNav1.8 mice demonstrated a markedly enhanced scratching response to nape

\section{Figure 2}

BRAFNav1.8 mice showed excessive spontaneous scratching and significantly enhanced pruritogen-evoked scratching. (A) BRAFNav1.8 mice developed age-dependent spontaneous scratching behavior from 6 to 14 weeks. $n=6$ to 8 per each time point. (B) BRAFNav1.8 mice developed skin lesions (arrows). (C) Prior to development of spontaneous scratching (at 4 6 weeks of age), BRAFNav1.8 mice showed significantly enhanced scratching responses to pruritogens injected into the nape (CQ; histamine [His]; compound 48/80 [48/80]; endothelin-1 [ET-1]) or to GRP injected i.t. Saline was the vehicle control. ${ }^{\star} P<0.05,{ }^{* \star *} P<0.001$, BRAFNav1.8 compared to WT littermates in the same test, BRAFNav1.8 vs. wild-type. $n=5-9$. intradermal (i.d.) injection of a number of pruritogens, including chloroquine (CQ), a nonhistaminergic pruritogen mediated in part through MRGPRA3 (27), histamine, compound 48/80, and endothelin-1 (Figure 2C). Scratching behavior elicited by intrathecal (i.t.) injection of GRP was also significantly enhanced (Figure 2C), suggesting augmented GRPR signaling. Therefore, both histaminergic and nonhistaminergic itch were significantly enhanced, indicating increased pruriceptive transmission from the periphery to the spinal cords of BRAFNav1.8 mice. To assess whether BRAF activation alters nociceptive processing, we carried out a series of pain behavioral tests prior to the onset of the spontaneous scratching. The responses of BRAF ${ }^{\text {Nav1.8 }}$ mice to either noxious or nonnoxious mechanical stimuli were normal (Supplemental Figure 4, A and B) as was acute thermal sensitivity (Supplemental Figure 4, C and D). In addition, BRAFNav1.8 mice exhibited normal licking and flinching responses to intraplantar injection of noxious chemicals such as formalin and mustard oil (Supplemental Figure 4, E and F). Thermal and mechanical hypersensitivity induced by intraplantar injection of capsaicin and complete Freund's adjuvant was also normal (Supplemental Figure 5, A-D). Moreover, BRAF ${ }^{\text {Nav1.8 mice }}$ exhibited normal motor function prior to the onset of excessive scratching behavior (Supplemental Figure 5E).

Massive ectopic expression of GRP/GRPR signaling in BRAF $F^{\text {Nav } 1.8}$ mice. The enhanced itch behavior prompted us to assess the expression of GRP in DRG neurons of BRAFNav1.8 mice. The number of GRP ${ }^{+}$ cells nearly doubled in BRAFNav1.8 mice $(13.5 \%)$ compared with that in wild-type mice (7.2\%) (Figure 3, A-C). Consistent with the observation of little variability in $\mathrm{pERK}^{+}$staining in DRGs or skin regions at different levels (Supplemental Figures 2 and 3), the percentage of $\mathrm{GRP}^{+}$cells detected was similar at different spinal segmental levels (Supplemental Figure 6). The proportion of cells expressing Mrgpra3, as detected by in situ hybridization (ISH), increased strikingly from $5.2 \%$ to $19.0 \%$ (Figure 3, D-F). The percentage of cells expressing histamine 1 receptor (H1R), a key receptor implicated in mediating histaminergic itch (28), increased from $12.7 \%$ to $20.0 \%$ (Figure 3, G-I). The ectopic expression of GRP, Mrgpra3, and H1R was found predominantly in TRPV1 ${ }^{+}$cells 

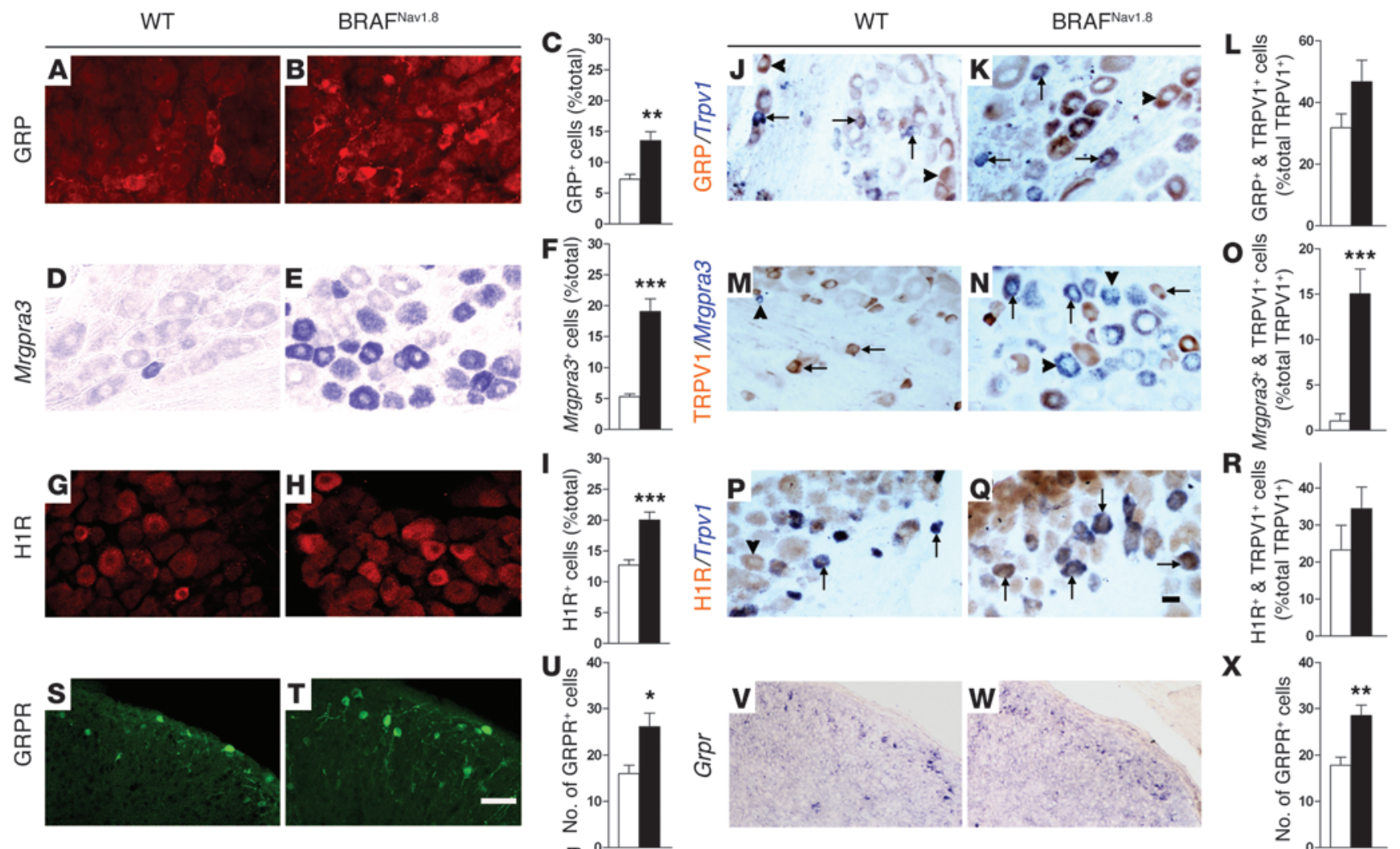

$\mathbf{X}$
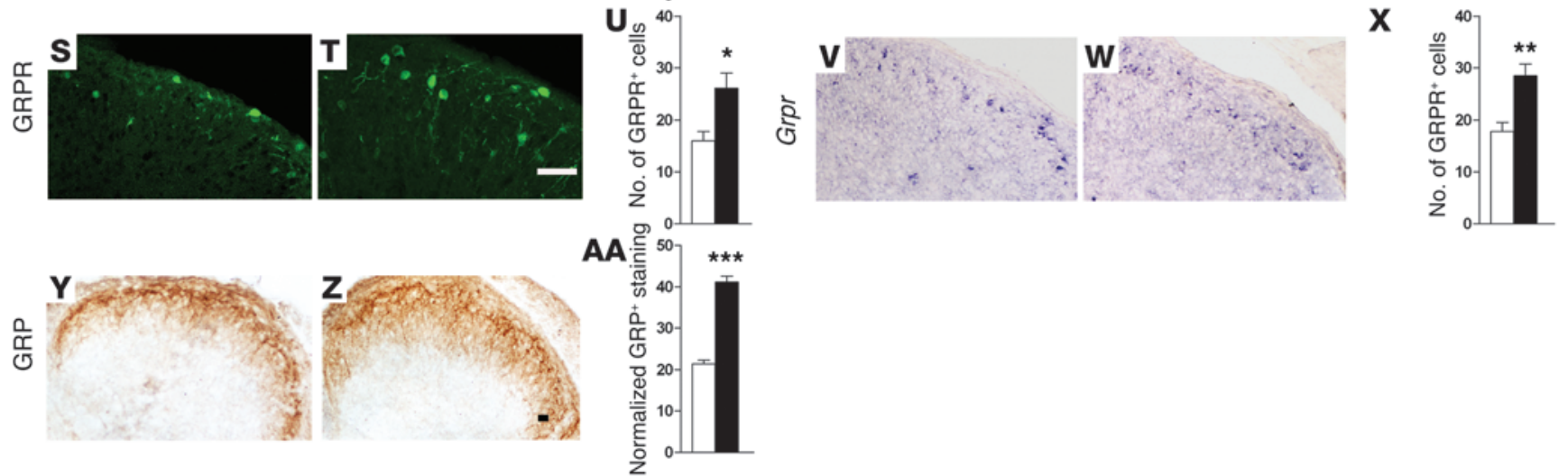

\section{Figure 3}

Ectopic expression of itch mediators in BRAFNav1.8 mice. (A-I) BRAFNav1.8 mice showed ectopic expression of (A-C) GRP, (D-F) Mrgpra3, and (G-I) H1R in DRGs. (J-R) BRAFNav1.8 mice showed increased expression of (M-O) Mrgpra3 (blue) but not (J-L) GRP (brown) and (P-R) H1R (brown) in Trpv1+ cells (blue in $\mathbf{J}-\mathbf{L}$ and $\mathbf{P}-\mathbf{R}$; brown in $\mathbf{M}-\mathbf{O}$ ). (S-X) Enhanced expression of GRPR as shown by (S-U) GFP staining and (V-X) Grpr mRNA in the dorsal horns of BRAFNav1.8 mice. (Y-AA) Enhanced GRP in primary afferents of BRAFNav1.8 mice. All mice were 8 12 weeks of age, except in V-AA, where 2-week-old mice were used. Scale bar: $20 \mu \mathrm{m}$. (J-Q) Arrows and arrowheads indicate TRPV1+ and TRPV1- neurons, respectively. ${ }^{\star} P<0.05,{ }^{\star \star} P<0.01,{ }^{\star \star \star} P<0.001$, BRAFNav1.8 mice vs. wild-type. $n=4$.

but also in TRPV1- cells (Figure 3, J-R); this was verified by their double staining with Trpv1 ISH (Figure 3, J-L and P-R) or TRPV1 antibody staining (Figure 3, M-O).

We next examined coexpression of Mrgpra3 with other markers in DRG neurons. The proportion of pERK/Mrgpra3-coexpressing cells was not significantly increased in BRAF ${ }^{N a v 1.8}$ mice (Supplemental Figure 7, A, B, and J). In wild-type mice, Mrgpra3 was mainly expressed in $\mathrm{P}^{2} \mathrm{X3}^{+} /$peripherin $^{+} / \mathrm{NF}^{200}{ }^{-} / \mathrm{CGRP}^{-}$cells (Supplemental Figure 7J). In BRAFNav1.8 DRG, despite the overall marked increase of $\mathrm{Mrgpra3}^{+}$cells, the expression pattern of Mrgpra3 in $\mathrm{P} \mathrm{X}^{+} /$peripherin $^{+} / \mathrm{NF}^{200}{ }^{-} / \mathrm{CGRP}^{-}$cells was unchanged. However, the proportion of $\mathrm{Mrgpra3}^{+}$cells coexpressing $\mathrm{CGRP}^{+}, \mathrm{H}^{+} \mathrm{R}^{+}$, and $\mathrm{GRP}^{+}$significantly increased (Supplemental Figure 7J). Moreover, the numbers of cells coexpressing Mrgpra3 with pERK, NF200, peripherin, P2X3, GRP, CGRP, and H1R all markedly increased in BRAFNav1.8 mice (Supplemental Figure $7 \mathrm{~K}$ ), indicating that
Mrgpra3 was ectopically expressed in several major types of DRG neurons, including both TRPV1 ${ }^{+}$and TRPV1- cells (Figure $3 \mathrm{~N}$ ), and most prominently in $\mathrm{GRP}^{+}$cells (Supplemental Figure $7 \mathrm{~K}$ ).

To examine GRPR expression, we took advantage of GRPR-EGFP mice that allow the detection of $\mathrm{GRPR}^{+}$neurons by anti-GFP staining. As expected, GFP expression was primarily restricted to lamina I of the spinal cords of GRPR-EGFP mice (Figure 3S), but its expression expanded entirely into lamina II of the spinal cords in GRPR-EGFP/BRAF ${ }^{\text {Nav1.8 }}$ mice (Figure 3T). This expression was validated by similar Grpr mRNA expansion detected by ISH (Figure 3, $\mathrm{V}$ and $\mathrm{W})$. Consistently, GRP ${ }^{+}$fibers were much more abundant and expanded into entire lamina II in BRAF ${ }^{\mathrm{Nav} 1.8}$ mice (Figure 3, $\mathrm{Y}-\mathrm{AA}$ ), matching the marked increase of GRP in DRG neurons (Figure 3, A-C). Notably, we did not observe an upregulation of GRP immunostaining intrinsic to dorsal horn interneurons of BRAF $^{\text {Nav1.8 }}$ mice relative to the control (Figure 3, Y-AA). 
A

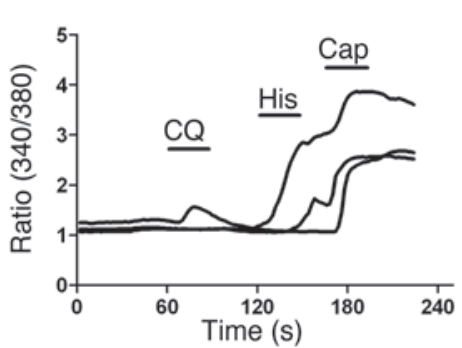

C

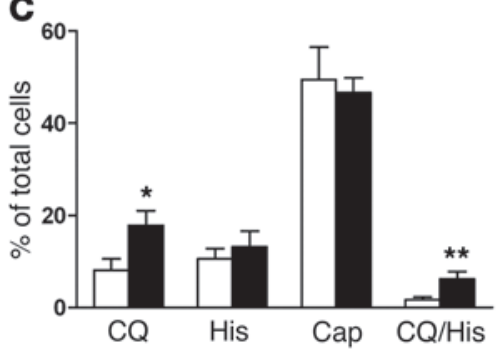

E

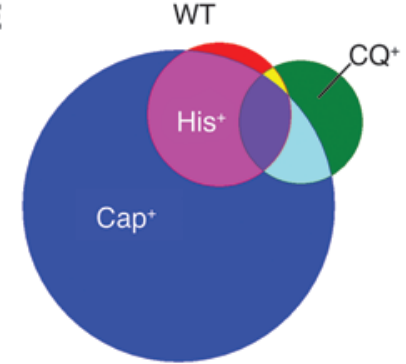

B

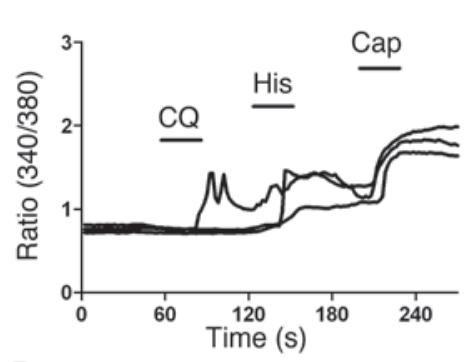

D

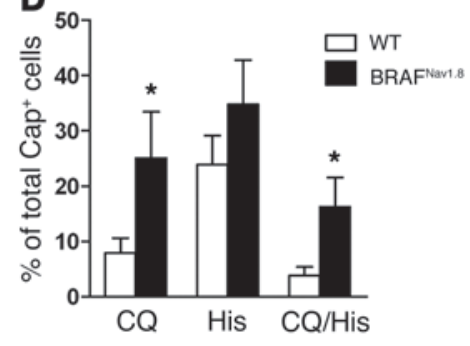

$\mathbf{F}$

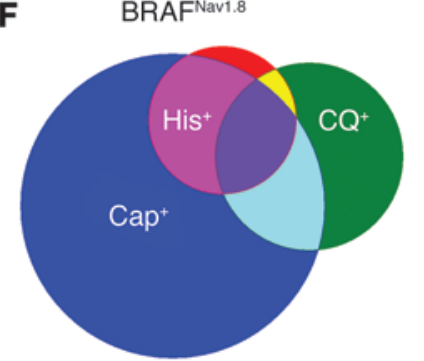

Figure 4

Enhanced and de novo responses to $C Q$ and histamine but not to capsaicin in DRG cells from BRAFNav1.8 mice. (A and $\mathbf{B}$ ) Three representative traces of DRG cells from (A) wildtype or (B) BRAFNav1.8 mice responsive to CQ $(100 \mu \mathrm{M})$, histamine $(50 \mu \mathrm{M})$, and capsaicin (Cap; $1 \mu \mathrm{M})$. (B) Note that one $C Q$-responsive cell tested also responded to histamine and capsaicin. (C) The percentage of CQ-responsive DRG cells, but not histamine- and capsaicin-responsive cells, was significantly increased in BRAFNav1.8 mice. More DRG cells responded to both $C Q$ and histamine in BRAFNav1.8 mice than in wild-type mice. (D) Of all capsaicin-sensitive cells in BRAFNav1.8 mice, there was a significant increase of cells that responded to $C Q$ or to both $C Q$ and histamine, whereas no difference was detected in the percentage of cells responsive to both capsaicin and histamine. ( $\mathbf{E}$ and $\mathbf{F}$ ) Venn diagrams showing the relative proportions of DRG cells of $(E)$ wild-type mice and (F) BRAFNav1.8 mice responsive to $C Q$, histamine, and capsaicin. ${ }^{*} P<0.05,{ }^{*} P<0.01$, BRAFNav1.8 vs. wild-type. $n=4$ (a total of 1,161 cells were tested).
Increased percentage of DRG neurons responding to CQ and histamine. We next analyzed the response of dissociated DRG neurons to CQ and histamine using calcium imaging. The percentage of cells responsive to CQ significantly increased in BRAFNav1.8 neurons (8.5\% wild-type vs. $19.4 \%$ BRAF $^{\text {Nav1 } 1.8}$ ), whereas that of histamineresponsive cells increased without statistical significance $(10.6 \%$ wild-type vs. 13.1\% BRAFNav1.8) (Figure 4, A-C). Few wild-type cells responded to both $\mathrm{CQ}$ and histamine, whereas there were significantly more cells responsive to both in BRAFNav1.8 neurons $(1.6 \%$ wild-type vs. 6.1\% BRAF ${ }^{\mathrm{Nav} 1.8}$ ) (Figure 4, A-C, E, and F). Of capsaicinresponsive cells, the percentage of cells responsive to CQ $(7.9 \%$

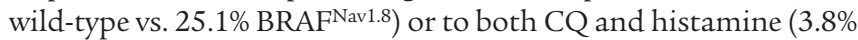
wild-type vs. 15.2\% BRAF ${ }^{\text {Nav1.8) }}$ ) increased significantly (Figure 4, D-F). Taken together, these data provide physiological evidence supporting de novo responsiveness of BRAF ${ }^{\text {av1. }}{ }^{2}$ DRG neurons to pruritogens but not to capsaicin.

Increased excitability of DRG neurons in response to bistamine and CQ. We next measured basic electrophysiological parameters of dissociated DRG neurons using current clamp recording. Among cells ranging in size from 17.5 to $32.5 \mu \mathrm{m}, 3$ firing patterns were observed: single spike (SS), delayed, and tonic firing (Figure 5, A-C, and Supplemental Table 1). Overall, SS cells showed a larger diameter, more hyperpolarized resting membrane potential, decreased input resistance, and higher rheobase values than delayed and tonic firing cells (Supplemental Table 1). Interestingly, the percentage of SS cells was much smaller in BRAF ${ }^{\mathrm{Nav} 1.8}$ mice compared with that in wild-type mice (36.3\% wild-type vs. $\left.16.3 \% \mathrm{BRAF}^{\mathrm{Nav}} 1.8\right)$, whereas the percentage of tonic firing cells almost doubled in
BRAF $^{\text {Nav1.8 }}$ mice (25.0\% wild-type vs. $47.5 \%$ BRAF Nav1.8) (Supplemental Table 1). The number of delayed firing cells remained the same (Supplemental Table 1). For either wild-type or BRAFNav1.8 mice, the cells responsive to pruritogens were typically tonic firing cells, which were readily excitable (Figure 5, D, E, G, and H). Only on rare occasion did SS cells respond to pruritogens. The tonic firing population of cells in BRAF ${ }^{\text {Nav1. }} .8$ mice exhibited a small increase in soma diameter, whereas SS neurons showed a smaller rheobase value relative to that of the control (Supplemental Table 1). The percentage of small-diameter cells responsive to histamine and CQ increased substantially in BRAF Nav1.8 mice (Figure 5, $\mathrm{F}$ and I). Despite this expansion, the response magnitudes, in terms of firing frequency in response to histamine or CQ, were largely the same (Figure 5, D, E, G, and H). Thus, there was a dramatic expansion of tonic firing cells at the expense of SS cells in tandem with increased expression of itch mediators. These changes might underlie the enhanced and spontaneous itch in BRAFNav1.8 mice.

Attenuated itch sensation of BRAF Nav1.8 mice by a blockade of histaminergic and GRP/GRPR signaling. To distinguish whether incessant scratching behavior was a result of aberrant projection of primary afferents or ectopic expression of itch mediators, we generated $\mathrm{Grp}^{-/-}$mice (Figure 6, A-C). Consistent with our previous observations (27), neither DRG neurons nor the spinal cords showed GRP staining in Grp ${ }^{-/-}$mice (Figure 6, D-G). A recent study claimed that GRP immunostaining is largely attributable to neurons intrinsic to the dorsal spinal cord (29). To clarify this, we repeated the dorsal rhizotomy experiment (12). As expected, on the ipsilateral side almost all CGRP ${ }^{+}$fiber staining in the dorsal horn 
A
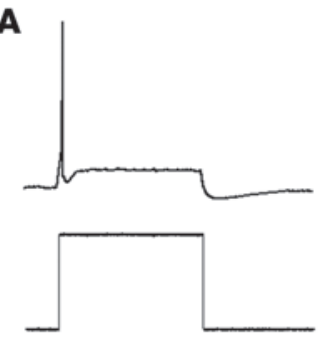

D

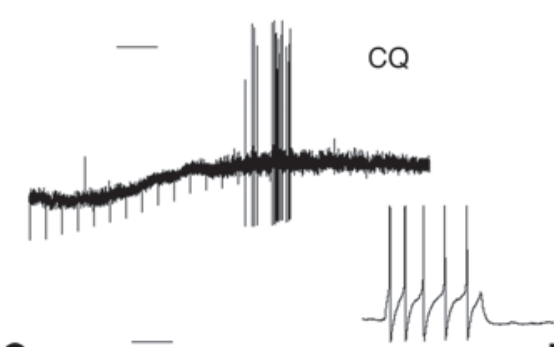

G

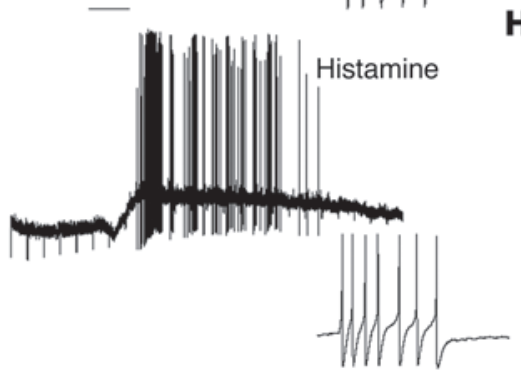

B
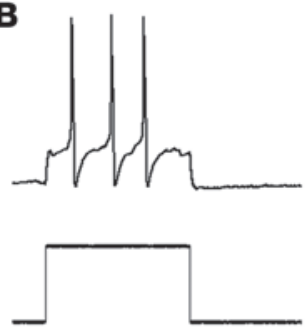

E

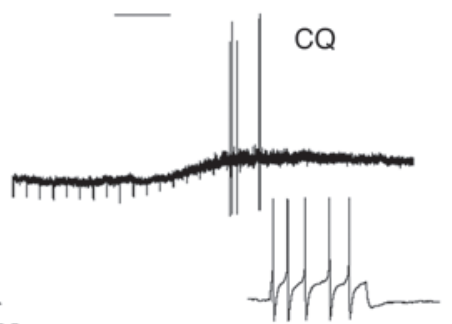

H

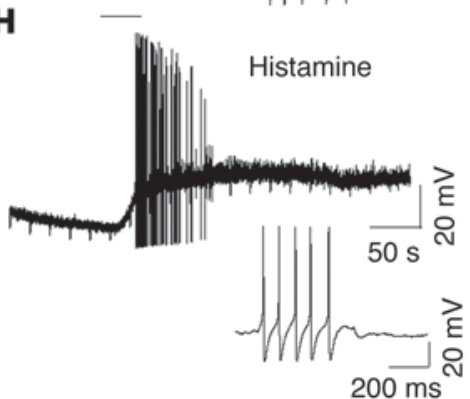

C

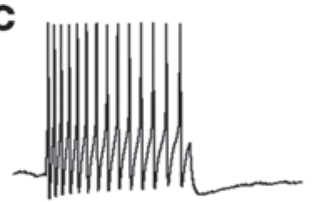

| $20 \mathrm{mV}$

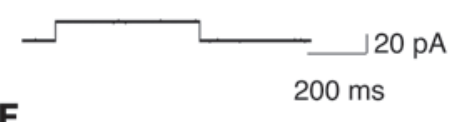

$\mathbf{F}$

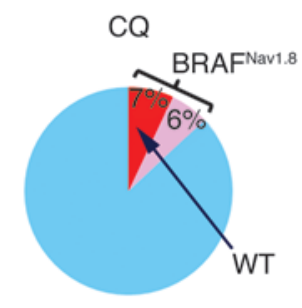

I

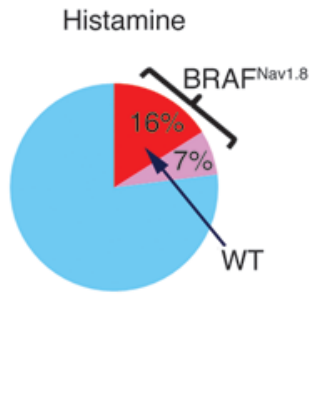

\section{Figure 5}

Electrophysiological characterization of DRG cells from BRAFNav1.8 mice and wild-type mice. (A-C) The BRAFNav1.8 DRG cells exhibited altered distribution of firing properties. In both BRAFNav1.8 mutant and wild-type cells, 3 firing patterns were routinely observed as (A) SS, (B) delayed, and $(\mathbf{C})$ tonic. (D, E, G, and $\mathbf{H}$ ) Representative action potential firing patterns of wild-type and BRAFNav1.8 cells in response to (D and $\mathbf{E}) \mathrm{CQ}_{\text {and }}$ $(\mathbf{G}$ and $\mathbf{H})$ histamine. The cells responding to pruritogens typically exhibited tonic firing properties with low rheobase values. Response magnitude was generally similar between wild-type and BRAFNav1.8 mice. ( $F$ and $\mathbf{I}$ ) Venn diagrams of total DRG cells showing that the percentage changes of DRG cells responsive to (F) CQ and (I) histamine substantially increased in BRAFNav1.8 mice relative to wild-type mice. (F) CQ (1 mM) induced action potential firing in 3 out of $44(6.8 \%)$ wild-type DRG cells and in 8 out of $61(13.1 \%)$ mutant DRG cells. (I) Histamine (100 $\mu$ M) induced action potential firing in 7 out of 44 (15.9\%) wild-type DRG cells and in 14 out of 61 (23.0\%) mutant DRG cells.

was lost, confirming the success of dorsal rhizotomy (Figure 6, $\mathrm{H}-\mathrm{J}$ and P). Similarly, GRP+ immunostaining was nearly abolished on the ipsilateral side of the surgery but remained intact on the contralateral side (Figure 6, K-P), confirming that the presence of $\mathrm{GRP}^{+}$immunostaining in the superficial dorsal horn was primary afferents dependent. These results directly challenge a previous study showing strong GRP staining in the spinal cord after the dorsal root rhizotomy (29). Importantly, spontaneous scratching of BRAF ${ }^{\mathrm{Nav1} 1.8}$ mice was markedly diminished in either $\mathrm{Grp}^{-/}$or Grpr KO mice and nearly abolished when $\mathrm{GRPR}^{+}$neurons were ablated with bombesin-saporin (Figure 7A and ref. 13). Scratching induced by i.t. GRP was also dramatically reduced after i.p. injection of 77427, a small-molecule GRP blocker (ref. 30 and Figure 7B). Neither Grp $p^{-/-}$nor Grpr KO mice showed an inhibitory effect of 77427 on CQ-elicited scratching observed in wild-type mice (Figure 7B), demonstrating the specificity of 77427 as a GRP blocker and supporting the role of GRP as an itch neurotransmitter in acute and chronic itch. Consistently, i.p. injection of 77427 significantly reduced spontaneous scratching in $\mathrm{BRAF}^{\mathrm{Nav1} 1.8}$ mice (Figure 7C) as did chlorpheniramine, a histamine antagonist (28), and U0126, a MEK inhibitor (31) (Figure 7C). U0126 failed to attenuate scratching behavior elicited by CQ and histamine (Figure 7D). Hence, these results demonstrate that ectopic ERK activation and expression of GRP and H1R are responsible for the spontaneous scratching of BRAF ${ }^{\mathrm{Nav} 1.8}$ mice.

Mice with chronic itch induced by allergic contact dermatitis and dry skin recapitulate ectopic ERK activation and expression of itch mediators in BRAF Nav1.8 mice. To assess the relevance of BRAF signaling to the development of chronic itch, we used a mouse model of allergic contact dermatitis (ACD), a prevalent skin disease triggered by direct skin contact with haptens or allergens, induced by topical application of a chemical hapten, 2, 4-dinitrofluorobenzene (DNFB) (32). A hallmark of ACD is intractable chronic itch that is resistant to antihistamines $(33,34)$. DNFB induces a $\mathrm{T}$ cell-mediated hypersensitivity reaction in human skin $(35,36)$ and similarly initiates sensitization and elicitation phases in mice that manifest 
A

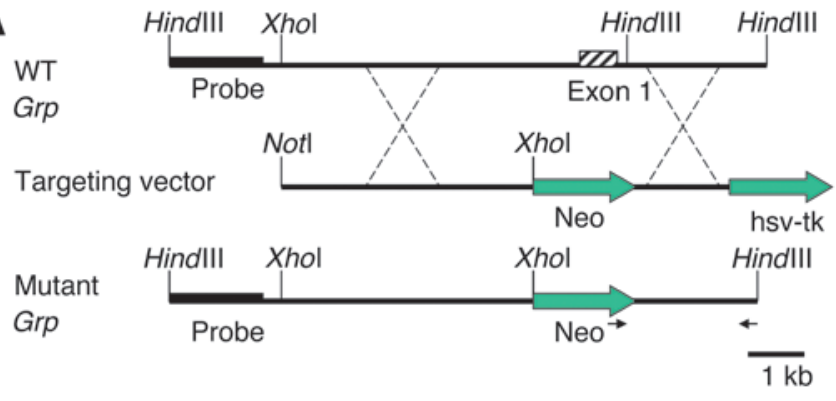

B
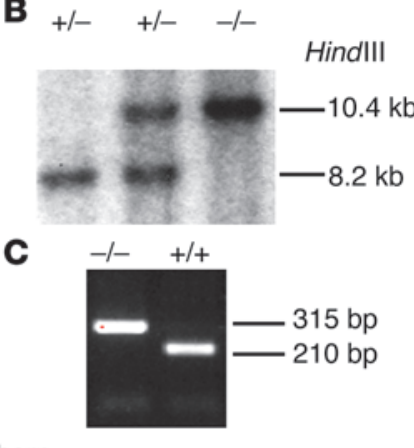

DRG
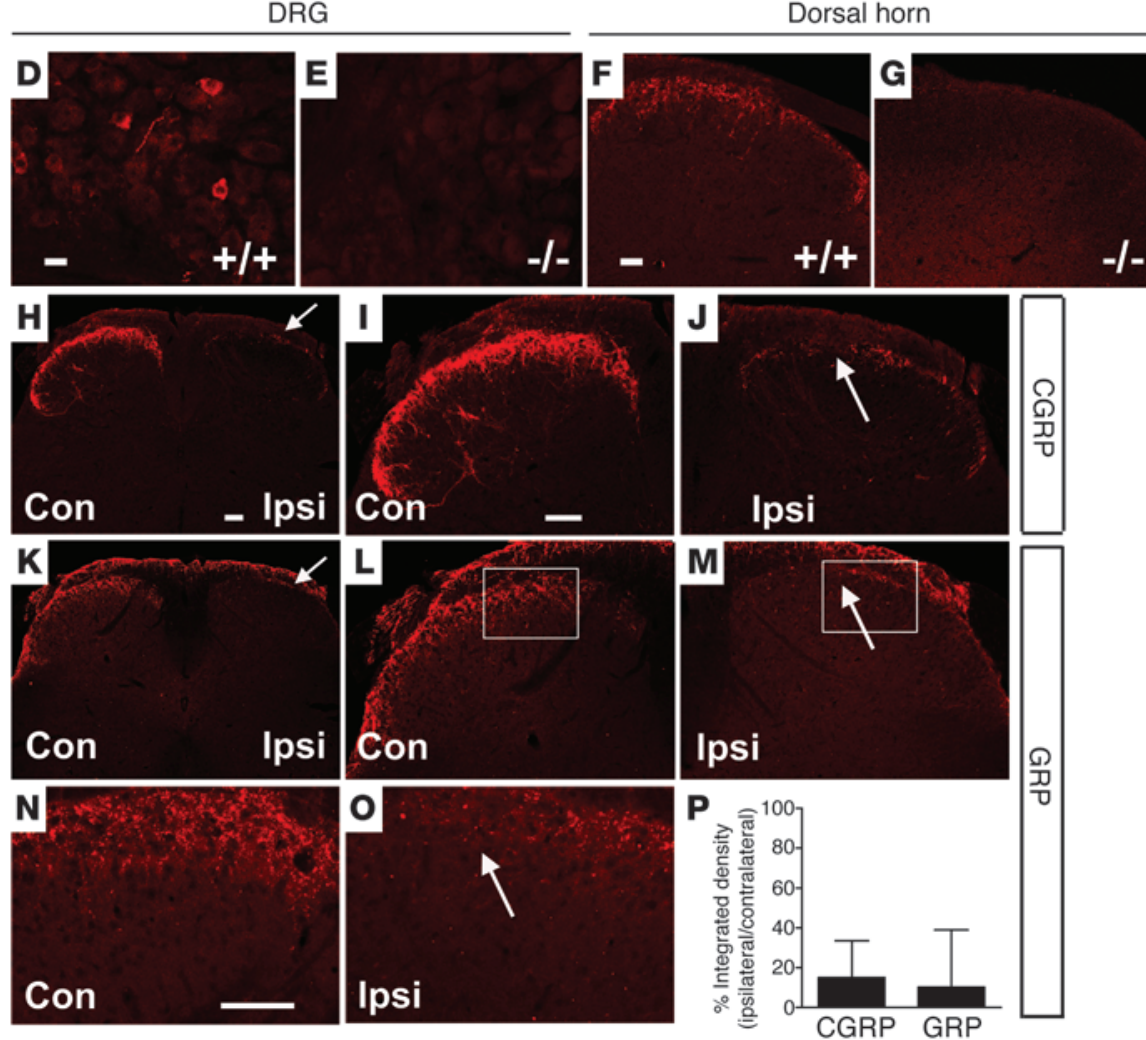

Figure 6

Generation of $\mathrm{Grp}^{-/-}$mice and confirmation of GRP expression in primary sensory neurons. (A) Targeting strategy for generation of $\mathrm{Grp}^{-1-}$ mice. (B and C) Germ line transmission was confirmed by (B) Southern blot and (C) PCR analysis. (D-G) GRP expression in the (D and $\mathbf{E})$ DRGs and ( $\mathbf{F}$ and $\mathbf{G}$ ) spinal cords of ( $\mathbf{D}$ and $\mathbf{F}$ ) wild-type mice and (E and $\mathbf{G}$ ) $\mathrm{Grp}^{-/-}$mice. (H-O) Expression of ( $\mathbf{H}-\mathbf{J})$ CGRP and $(\mathbf{K}-\mathbf{O})$ GRP in the lumbar spinal cords of C57BL/6J mice 14 days after unilateral dorsal rhizotomy (L5). On contralateral sides, both ( $\mathbf{H}$ and $\mathbf{~ I})$ $\mathrm{CGRP}^{+}$and $(\mathbf{K}, \mathbf{L}$, and $\mathbf{N}) \mathrm{GRP}^{+}$ fibers were mainly in the superficial dorsal horn (lamina I, Ilo); but on the ipsilateral sides, both ( $\mathbf{H}$ and $\mathbf{J})$ CGRP and $(\mathbf{K}, \mathbf{M}$, and O) GRP staining was lost after unilateral L5 dorsal rhizotomy. Arrows indicate the elimination of staining. $n=3$ per group. (P) Quantitation of remaining CGRP+ $(15.1 \%)$ and GRP+ $(10.2 \%)$ staining in the L5 superficial dorsal horn after rhizotomy. Boxed areas in $\mathbf{L}$ and $\mathbf{M}$ are shown at higher magnification in $\mathbf{N}$ and $\mathbf{O}$. Scale bar: $10 \mu \mathrm{m}$ (D and $\mathbf{E}) ; 20 \mu \mathrm{m}$ ( $\mathbf{F}$ and $\mathbf{G}$ ); $40 \mu \mathrm{m}(\mathrm{H}-\mathbf{O})$. as spontaneous scratching behavior (ref. 37 and see below). DNFB treatment resulted in thickening of epidermis, increased lymph node and spleen size, increased number of neutrophils, infiltration of inflammatory mast cells, and elevated serum IgE levels (Figure 8, A-E). The number of white blood cells also increased, but the difference was not statistically significant (Figure 8C). Thymic stromal lymphopoietin (TSLP), a marker for skin inflammation and dermatitis (38), also increased significantly (Figure 8F). Importantly, DNFB-induced spontaneous scratching was significantly diminished by i.t. U0126 (Figure 9C). In mice treated with DNFB, ectopic ERK activation and expression of GRP and Mrgpra3 were detected in DRG neurons (Figure 9, D-L), and increased GRPR expression was detected in the spinal cord (Figure 9, M-O).

Finally, we analyzed whether BRAF signaling may have a role in itch induced by skin dryness (xerosis). Dry skin is caused by disrupting cutaneous barrier function and is associated with rough, scaly, and flaky skin often accompanied by chronic pruritus (39). Topical application of an acetone/ether mixture followed by water leads to spontaneous scratching (Figure 9B), resulting from skin stratum corneum dehydration and epidermal water loss, and dry skin itch is mediated by histamine-independent mechanisms $(40,41)$. Similarly, dry skin itch was attenuated by U0126 (Figure 9C). Ectopic ERK activation and expression of GRP, Mrgpra3, and GRPR were observed in DRG neurons of mice with dry skin itch (Supplemental Figure 8). To assess whether spinal ERK activation is required to maintain spontaneous itch, we examined ERK activation in the dorsal horns of mice 1 day after the last DNFB or dry skin treatment. For a direct comparison of pERK activation to central hypersensitivity, pERK staining after intraplantar capsaicin injection (a model of inflammatory pain) was used as the control (ref. 42 and Supplemental Figure 9). We did not detect pERK ${ }^{+}$ cells in the spinal cord, despite ongoing spontaneous itch in both chronic itch models (Supplemental Figure 9), indicating that pERK upregulation in DRG instead of the spinal cord is necessary and sufficient for maintaining both peripheral and central sensitization. Interestingly, $\mathrm{CGRP}^{+}$and $\mathrm{IB}^{+}$fibers projected normally in the dorsal horns of mice with ACD and dry skin itch (Supplemental Figure 10), unlike the expansion of peptidergic and nonpeptidergic 
A
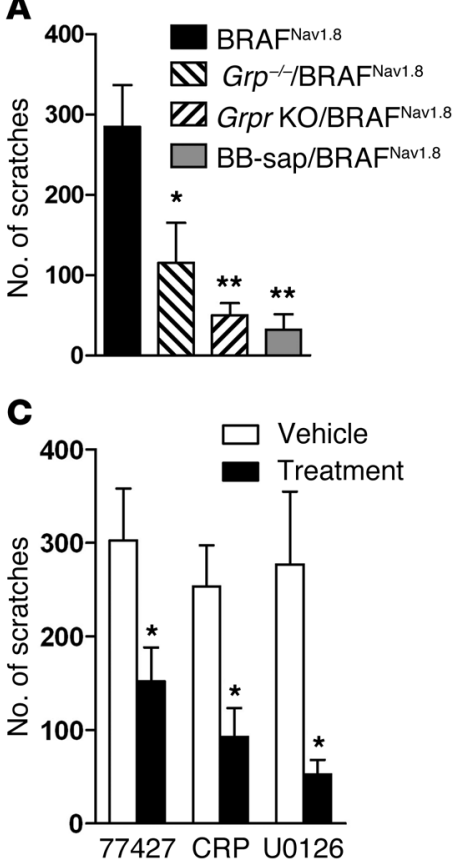

B

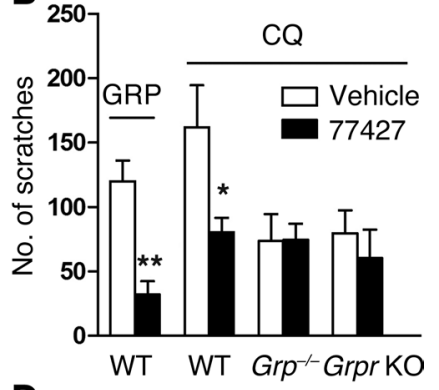

D

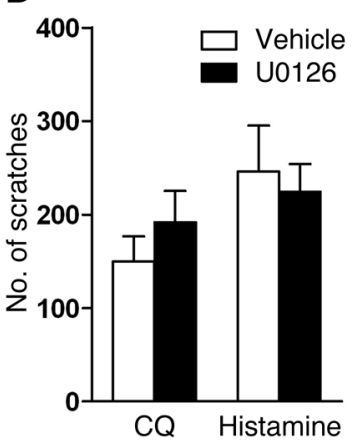

Figure 7

Genetic and pharmacologic blockade of spontaneous scratching behavior in BRAFNav1.8 mice. (A) Spontaneous scratching of BRAFNav1.8 mice was significantly attenuated in $\mathrm{Grp}^{-/-}$or $\mathrm{Grpr}$ $\mathrm{KO}$ background and in mice treated with i.t. bombesin-saporin (BB-sap). ${ }^{*} P<0.05,{ }^{*} P<0.01,1$-way ANOVA followed by posthoc analysis, wild-type vs. BRAFNav1.8 mice. (B) GRP blocker 77427 (i.p.) significantly reduced scratching elicited by GRP (i.t.) or $\mathrm{CQ}$ (i.d.) in wild-type mice but not in $\mathrm{Grp}^{-/-}$or Grpr KO mice. (C) Spontaneous scratching of BRAFNav1.8 mice was significantly reduced by i.p. injection of 77427 or the H1R antagonist chlorpheniramine (CRP) or i.t. injection of a MEK inhibitor, U0126. (D) Scratching behavior of mice induced by i.d. CQ and histamine did not differ after i.t. U0126. ${ }^{\star} P<0.05,{ }^{* *} P<0.01,2$-tailed $t$ test vs. vehicle controls. $n=6-10$. fibers seen in BRAFNav1.8 mice, indicating that the latter was due to developmental changes and unlikely to contribute to the chronic itch behavior phenotypes. Taken together, these results reveal that, in mouse models of ACD and dry skin itch, ectopic ERK activation and expression of GRP and Mrgpra3 occurred similarly to that in BRAF $^{\text {Nav1.8 }}$ mice and suggest that $\mathrm{pERK}$ and GRP/GRPR signaling are critical for the progression and maintenance of long-lasting itch transmission (Figure 9P).

\section{Discussion}

Here, we identify the BRAF pathway as an upstream regulator of expression of a cohort of itch mediators in mouse sensory neurons. Our data further demonstrate that GRP/GRPR signaling plays an important role in the initiation and maintenance of long-lasting itch in 3 distinct genetic and/or clinically relevant chronic itch models. Finally, our data suggest that a phenotypic switch from nociceptors to pruriceptors underlies the transition from acute itch signaling to pathological itch state.

BRAFNav1.8 mice are a new and unique genetic model for unraveling how pruriceptive transmission is progressively enhanced at the molecular and circuit levels, as increasing scratching behavior correlates well with an expression profile change of itch mediators. The fact that enhanced evoked scratching preceded the manifestation of spontaneous itch (from 3 weeks of age to around 6 weeks of age) suggests the existence of a postnatal period for developmental maturation of itch circuitry as well as a threshold for the intensity of ongoing itch signaling. We cannot exclude the possibility that skin lesion or inflammation resulting from excessive scratching might have contributed to further increase of scratching as mice age. Interestingly, we have observed more $\mathrm{pERK}^{+}$fiber innervation in the skin. Coupled with increased $\mathrm{GRP}^{+}$fiber innervation in skin of mice with atopic dermatitis (43), it will be interesting to see whether the interactions between skin lesion/inflammation and enhanced itch-specific nerve innervation are part of positive feedback mechanisms that are engaged to provoke more itching signaling and exacerbate scratching response. In both ACD and dry skin models, similar progressively increased scratching responses were observed, albeit in a much shorter period, suggesting that skin sensitization/inflammation may account for at least some aspects of peripheral and central sensitization of itch signaling.

Because the total number of sensory neurons remained unaltered, the finding that substantial de novo expression of GRP and Mrgpra3 primarily concentrated in TRPV $1^{+}$neurons implies that a small subset of nociceptors has acquired novel pruriceptive properties. Similar de novo neuronal responsiveness to pruritogens has been observed in mice with dry skin (41). Our finding that a population of tonic firing sensory neurons was significantly expanded at the expense of SS cells indicates that some nociceptors switched from unresponsive to responsive or excitable to pruritogenic stimuli. We refer to cells that express in situ itch mediators as "primary pruriceptors," which form the basal circuits for acute itch transmission, while cells expressing de novo itch mediators are "secondary pruriceptors." Mechanistically, we suggest that a recruitment of secondary pruriceptors is a prerequisite for initiating and sustaining prolonged pruriceptive transmission. This phenotypic switch mechanism may also underlie central sensitization of itch in the context of chronic itch.

Our studies reveal that enhanced and persistent expression of GRP in sensory neurons is necessary for transmitting itch-specific information to the spinal cord in chronic itch states. A sustained release of GRP may necessitate additional GRPR signaling or the secondary itch neurons, so that the capacity of the central itch circuit is expanded to accommodate the amplified itch signaling to the brain. It remains unclear, however, which subpopulation of dorsal horn neurons is capable of expressing de novo GRPR. The present study confirms the specificity of the anti-GRP antibody $(27,29)$ as well as primary afferents as the source of GRP protein in the superficial dorsal horn, which was first demonstrated in rats (44). While it has been known that Grp mRNA is expressed in the dorsal horn $(45,46)$, importantly, chronic itch did not upregulate 

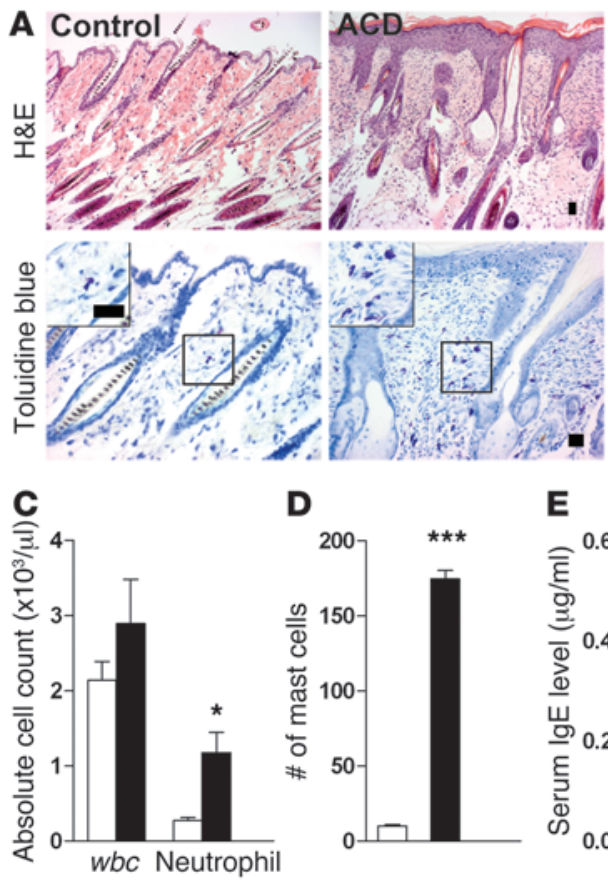

D

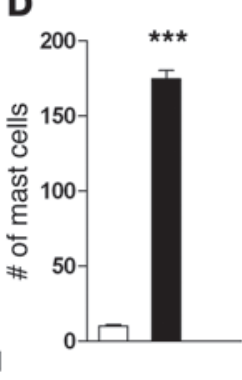

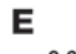

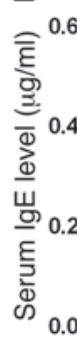

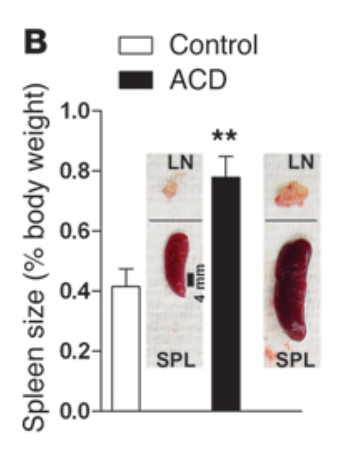

Figure 8

Analysis of skin and immune system in adult mice with ACD. (A) H\&E and toluidine blue staining of the neck skin of the mice with ACD and the control mice. The mice with ACD showed epidermal hyperplasia, hyperkeratosis, acanthosis, dermal inflammation, and mast cell infiltration (insets show a few mast cells). Scale bar: $25 \mu \mathrm{m}$. (B and C) The systemic manifestations of allergic dermatitis were presented as highlighted by their (B) enlarged spleen and lymph nodes and (C) significantly increased blood neutrophil counts. SPL, spleen. Scale bar: $4 \mathrm{~mm}$. (D) Quantitative analysis confirmed that ACD enhanced the extent of mast cell infiltration in the dermis. Mast cells in 9 random $\times 200$ microscope fields were counted and averaged. (E) IgE levels were highly elevated in mice with ACD. (F) Ts/p mRNA was overexpressed in the ACD skin. Representative RT-PCR images (bottom). ${ }^{*} P<0.05,{ }^{* *} P<0.01,{ }^{* * *} P<0.001$, mice with ACD vs. the controls (acetone only). $n=6$.
GRP protein in spinal interneurons. The corroborative evidence, therefore, led us to conclude that spinal Grp mRNA does not equate to GRP protein expression. Failure to detect Grp mRNA in DRG neurons by ISH or lack of EGFP in DRGs of GRP-EGFP transgenic mice highlights the technical limitations that have resulted in differing interpretation $(29,47)$.

Complementary to our loss-of-function studies $(12,13)$, the finding that a dramatic upregulation of GRP/GRPR signaling failed to impact both acute and chronic pain behavior of BRAFNav1.8 mice in a profound manner reinforces the idea that GRP/GRPR signaling represents a dedicated molecular labeled line for itch from periphery to the spinal cord. This is in contrast to B-type natriuretic peptide (BNP), which has recently been proposed to be dedicated to activation of its receptor natriuretic peptide receptor-A (NPRA) for itch transmission from DRGs to the spinal cord (47). However, BNP-NPRA-mediated autoregulation in DRG neurons was also implicated in nociceptive processing in DRG neurons (48). Indeed, we found that i.t. injection of BNP also regulated inflammatory pain behavior (Zhao et al., unpublished observations).

$\mathrm{BRAF}^{\mathrm{Nav1.8}}$ mice enable us to position BRAF signaling upstream of key itch effectors in sensory neurons of mice with chronic itch. Notably, unlike DRG neurons, pERK activation in the spinal cords of mice with ACD and dry skin occurred only transiently after skin was acutely sensitized and was absent the following day when spontaneous scratching was persistent. This suggests that pERK activation in the spinal cord is dispensable for maintaining central sensitization of itch. Nonetheless, the capability of the BRAF signaling cascade to regulate expression of a wide range of itch mediators from histaminergic itch to nonhistaminergic itch genes reveals a remarkable molecular and cellular specificity as well as plasticity intrinsic to sensory neurons.

ERK signaling was differentially activated in nociceptors in response to varying noxious stimuli $(49,50)$. While it is unclear why pruriceptive genes are selectively activated, our analyses of
ACD and dry skin models suggest that different types of persistent pruriceptive stimuli may differentially trigger pERK signaling in different subsets of pruriceptors. For example, although ERK was activated, no upregulation of H1R was observed in ACD or dry skin models. Moreover, despite ERK activation and ectopic expression of GRP in DRG neurons and GRPR in the spinal cord, the temporal and spatial patterns of spontaneous scratching between these chronic itch models differ dramatically. Thus, behavioral output encoded by the sustained ERK activation in sensory neurons may be dictated by the nature of a pruriceptive stimulus as well as the state of itch or pain sensitization. A combinatorial activation of itch mediators and types of neurons in which they are activated is manifest in the duration, intensity, and regional patterns of itch response, the last of which is also inherently restrained by the areas in which a mouse can reach and scratch using its hind limbs.

How is ERK signaling triggered upon acute contact dermatitis or dry skin induction? A host of extracellular stimuli can activate ERK signaling $(21,22,51)$, making it possible that heightened levels of such factors in the extracellular milieu as a result of local metabolic dysregulation or inflammation can stimulate intracellular BRAF to augment itch signaling. Considering the progressive development of chronic itch, it is possible that these factors may also activate a diverse group of GPCRs, which in turn initiates the RAF/MEK/ERK cascade $(21,52)$. As a result, an amplified BRAF signal may further sensitize itch circuits through a positive feedback loop via GPCR-dependent integration of downstream ERK signaling. Identification of ERK-dependent transcriptional events would be a logical next step to link extracellular stimuli to the expression of downstream effector genes that encode itch information. Finally, the present study supports the role of GRP as an itch-specific neuropeptide in sensory neurons, in agreement with previous findings that GRP was upregulated in primary afferents of primates with chronic itch (53) and in patients (54). 

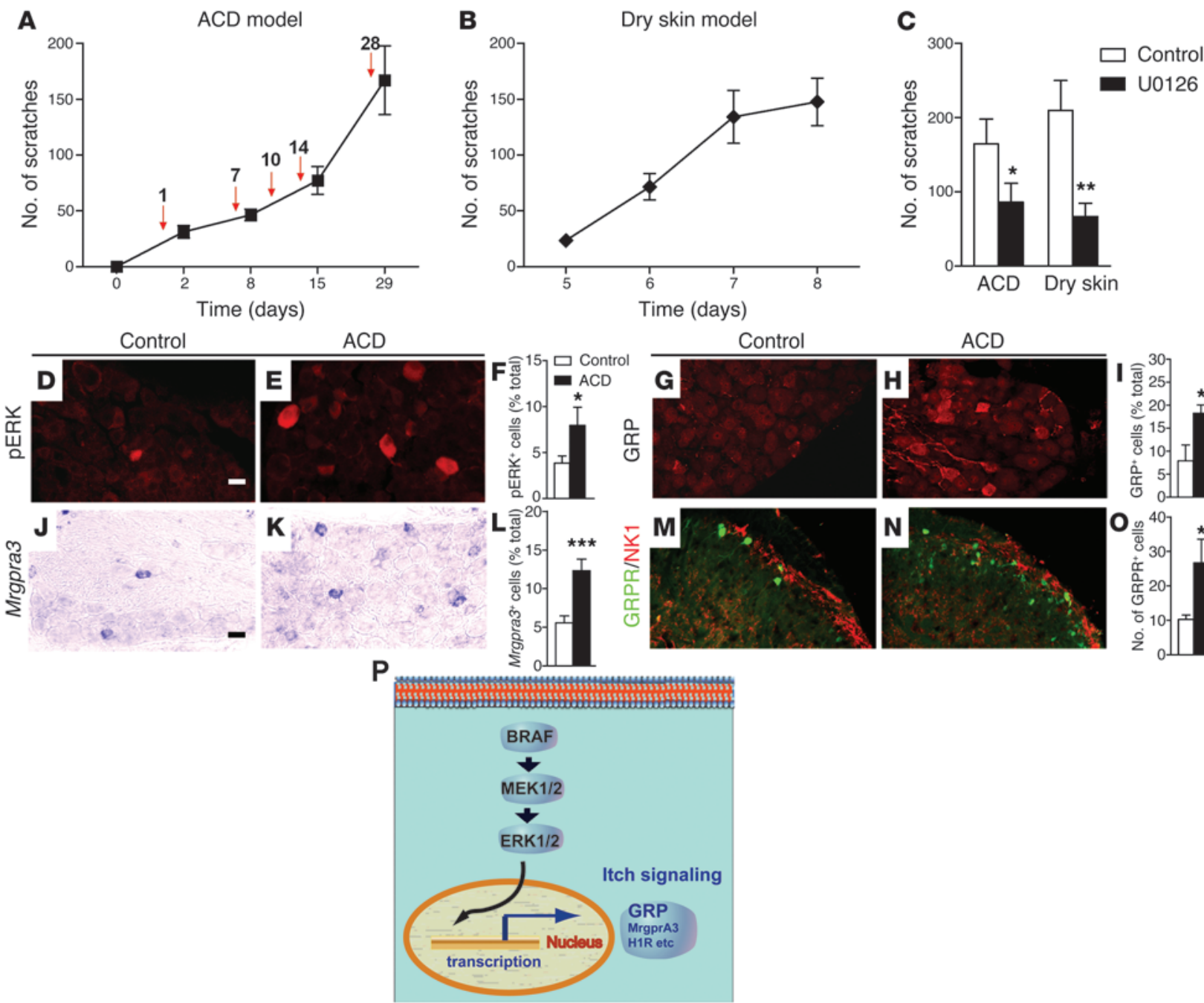

\section{Figure 9}

Mice with ACD- and dry skin-induced itch recapitulate the phenotype of BRAFNav1.8 mice. (A) Mice with ACD induced by DNFB exhibited progressively increased scratching behavior. Red arrows indicate the time points when DNFB was applied, and spontaneous scratches were counted 24 hours afterward. $n=7$. (B) Progressively increased scratching behavior was induced in mice with dry skin itch. $n=13$. (C) Spontaneous scratching of mice with ACD or dry skin was significantly reduced by i.t. U0126. $n=5 \sim 9$ per group. ${ }^{\star} P<0.05$, ${ }^{\star *} P<0.01$, mice treated with U0126 vs. the controls. (D-F) Ectopic activation of ERK and (G-I) expression of GRP. (J-L) Mrgpra3 in DRGs and (M-O) GRPR as shown by GFP staining (E, H, $\mathbf{K}$, and $\mathbf{N}$ ) in the dorsal horns of mice with ACD compared to ( $\mathbf{D}, \mathbf{G}, \mathbf{J}$, and $\mathbf{M})$ the controls (acetone only). In $\mathbf{M}-\mathbf{0}$, GRPR-EGFP mice were used. Results in $\mathbf{F}, \mathbf{I}, \mathbf{L}$, and $\mathbf{O}$ are quantitative comparisons between 2 groups $(n=3$ per group). Scale bar: $10 \mu \mathrm{m}(\mathbf{D}, \mathbf{E}, \mathbf{G}$, and $\mathbf{H}) ; 20 \mu \mathrm{m}(\mathbf{J}, \mathbf{K}, \mathbf{M}$, and $\mathbf{N})$. (P) Diagram illustrates BRAF/MEK/ERK regulation of itch molecular phenotype in DRG. ${ }^{\star} P<0.05,{ }^{\star \star \star} P<0.001$, mice with ACD vs. the controls.

We suggest that an upregulation of GRP/GRPR represents a hallmark of the development of chronic itch. A repertoire of pharmacologic inhibitors of the BRAF cascade in clinical trials for patients with cancer (55) may be used in combination with inhibitors of GRP or GRPR to provide a more effective strategy for ameliorating chronic itch conditions than either type of inhibitor alone.

\section{Methods}

Additional details regarding this study are provided in the Supplemental Methods

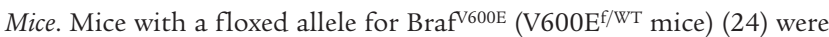
crossed with Nav1.8 $\mathrm{Cre} /+$ mice (25) to generate mice with specific expression of $\mathrm{Braf}^{\mathrm{V} 600 \mathrm{E}}$ in Nav1.8 ${ }^{+}$cells (referred to herein as BRAFNav1.8 mice). Littermates harboring the genotypes Nav1.8 ${ }^{\mathrm{WT} / \mathrm{WT}}$ with Braf ${ }^{\mathrm{V} 600 \mathrm{E}}$, Nav1.8 $8^{\mathrm{Cre} / \mathrm{WT}}$ with $\mathrm{Braf}^{\mathrm{WT} / \mathrm{WT}}$, and Nav1.8 ${ }^{\mathrm{WT} / \mathrm{WT}}$ with Braf ${ }^{\mathrm{WT} / \mathrm{WT}}$ were used as controls. Generation of Grpr KO mice and GRPR-EGFP mice was described previously $(12,56,57)$. For generation of $\mathrm{Grp}^{-1-}$ mice, see the Supplemental Methods.

Behavioral studies. The motor, itch, and pain behavioral studies were performed as described previously $(12,13)$. Chronic itch models were used as described previously $(40,58)$.

Immunohistochemistry and ISH. Standard procedures were used as described previously (59).

RT-PCR and Western blot analysis. RT-PCR and Western blot analysis were carried out as described previously (11)

Calcium imaging and electrophysiology. Dissociated DRG neurons were loaded with fura 2-acetoxymethyl ester (Invitrogen) and imaged at 340 and $380 \mathrm{~nm}$ 
excitation to detect intracellular-free calcium for analysis of the relative proportions of DRG neurons responsive to pruritogens. Current clamp recordings were acquired on dissociated DRG neurons as described previously (60).

Skin and immune system in mice with $A C D$. Back neck skin and blood samples were harvested from mice for various analyses as described previously (61).

Statistics. Behavioral tests, cell counting, or molecular analysis were performed by observers blinded to the treatments and/or genotypes of the animals used. Unless indicated otherwise, cervical DRGs and dorsal horns were used. All quantification data are presented as mean \pm SEM, and error bars represent SEM. Statistical comparisons were performed with 1-way or 2 -way ANOVA followed by post-hoc analysis, Student's $t$ test, or the $\chi^{2}$ test, as specifically indicated in the figure legends. However, unless otherwise indicated, the statistical analyses were done using $t$ test, and $P<0.05$ was considered statistically significant.

Study approval. All experiments were performed in accordance with the guidelines of the NIH and the International Association for the Study of Pain and were approved by the Animal Studies Committee at Washington University School of Medicine and the Institutional Animal Care and Use Committees at Weill Cornell Medical College.

\section{Acknowledgments}

We thank Q. Li, J. Yin, L.R. Han, L. Zhou, A. Sure, and M. Giger for genotyping, behavioral test, and technical support; Z.Q. Xie for help with a chronic itch model; and X. Jian for help developing $\mathrm{Grp}^{-/-}$mice. We also thank J. Wood for Nav1.8-cre mice, C. Pritchard and R. Marais for Braf ${ }^{\mathrm{V} 600 \mathrm{E}}$ mice, and Q. Liu, M. Jacquin, G. Giesler, and lab members for comments. J. Jeffry has been supported by a NIDA training grant. The research was supported by RO1 grants from the National Institute of Arthritis and Musculoskeletal and Skin Diseases to Z.-F. Chen (RO1 AR056318-01A1) and the National Eye Institute (1R01EY022409 and administrative supplement 3R01EY02240901S1), the Whitehall Foundation (2010-08-61) to J. Zhong, and a NIH RO1 grant to R. Kopan (R01DK066408).

Received for publication April 17, 2013, and accepted in revised form August 12, 2013.

Address correspondence to: Zhou-Feng Chen, Center for the Study of Itch, Department of Anesthesiology, 8054 Mail Stop, Washington University School of Medicine, 600 S. Euclid Ave., St. Louis, Missouri 63110, USA. Phone: 314.747.5093; Fax: 314.362.8571; E-mail: chenz@wustl.edu. Or to: Jian Zhong, Burke Medical Research Institute, Weill Medical College of Cornell University, 785 Mamaroneck Ave., White Plains, New York 10605, USA. Phone: 914.368.3132; Fax: 914.597.2225; E-mail: jiz2010@med.cornell.edu.

Seungil Kim's present address is: Department of Cell and Tissue Biology, Craniofacial and Mesenchymal Biology, UCSF, San Francisco, California, USA.

Zhong-Chun Liu's present address is: Department of Psychiatry, Renmin Hospital, Wuhan University, Wuhan, China.
1. Imamachi $\mathrm{N}$, et al. TRPV1-expressing primary afferents generate behavioral responses to pruritogens via multiple mechanisms. Proc Natl Acad Sci U S A. 2009; 106(27):11330-11335.

2. Andrew D, Craig AD. Spinothalamic lamina I neurons selectively sensitive to histamine: a central neural pathway for itch. Nat Neurosci. 2001; 4(1):72-77.

3. Davidson S, Giesler GJ. The multiple pathways for itch and their interactions with pain. Trends Neurosci. 2010;33(12):550-558.

4. Ross SE. Pain and itch: insights into the neural circuits of aversive somatosensation in health and disease. Curr Opin Neurobiol. 2011;21(6):880-887.

5. Jeffry J, Kim S, Chen ZF. Itch signaling in the nervous system. Physiology (Bethesda). 2011;26(4):286-292.

6. McNeil B, Dong X. Peripheral mechanisms of itch. Neurosci Bull. 2012;28(2):100-110.

7. Han SK, Simon MI. Intracellular signaling and the origins of the sensations of itch and pain. Sci Signal.2011; 4(185):pe38.

8. Shim WS, et al. TRPV1 mediates histamine-induced itching via the activation of phospholipase A2 and 12-lipoxygenase. J Neurosci. 2007;27(9):2331-2337.

9. Wilson SR, et al. TRPA1 is required for histamine-independent, Mas-related G protein-coupled receptor-mediated itch. Nat Neurosci. 2011; 14(5):595-602.

10. Han L, et al. A subpopulation of nociceptors specifically linked to itch. Nat Neurosci. 2013;16(2):174-182.

11. Liu XY, et al. Unidirectional cross-activation of GRPR by MOR1D uncouples itch and analgesia induced by opioids. Cell. 2011;147(2):447-458.

12. Sun YG, Chen ZF. A gastrin-releasing peptide receptor mediates the itch sensation in the spinal cord. Nature. 2007;448(7154):700-703.

13. Sun YG, Zhao ZQ, Meng XL, Yin J, Liu XY, Chen ZF. Cellular basis of itch sensation. Science. 2009; 325(5947):1531-1534.

14. Ikoma A, Steinhoff M, Stander S, Yosipovitch G, Schmelz M. The neurobiology of itch. Nat Rev Neurosci. 2006;7(7):535-547.
15. Paus R, Schmelz M, Biro T, Steinhoff M. Frontiers in pruritus research: scratching the brain for more effective itch therapy. J Clin Invest. 2006; 116(5):1174-1186.

16. Ross SE, et al. Loss of inhibitory interneurons in the dorsal spinal cord and elevated itch in Bhlhb5 mutant mice. Neuron. 2010;65(6):886-898.

17. Lagerstrom MC, et al. VGLUT2-dependent sensory neurons in the TRPV1 population regulate pain and itch. Neuron. 2010;68(3):529-542.

18. Liu Y, et al. VGLUT2-dependent glutamate release from nociceptors is required to sense pain and suppress itch. Neuron. 2010;68(3):543-556.

19. Wilson SR, et al. The ion channel TRPA 1 is required for chronic itch. J Neurosci. 2013;33(22):9283-9294.

20. Han L, et al. A subpopulation of nociceptors specifically linked to itch. Nat Neurosci. 2013;16(2):174-182.

21. Pearson G, et al. Mitogen-activated protein (MAP) kinase pathways: regulation and physiological functions. Endocr Rev. 2001;22(2):153-183.

22. Chong H, Vikis HG, Guan KL. Mechanisms of regulating the Raf kinase family. Cell Signal. 2003; 15(5):463-469.

23. Zhong J, Li X, McNamee C, Chen AP, Baccarini $\mathrm{M}$, Snider WD. Raf kinase signaling functions in sensory neuron differentiation and axon growth in vivo. Nat Neurosci. 2007;10(5):598-607.

24. Mercer K, et al. Expression of endogenous oncogenic V600EB-raf induces proliferation and developmental defects in mice and transformation of primary fibroblasts. Cancer Res. 2005; 65(24):11493-11500.

25. Stirling LC, et al. Nociceptor-specific gene deletion using heterozygous NaV1.8-Cre recombinase mice. Pain. 2005;113(1-2):27-36.

26. Caterina MJ, Schumacher MA, Tominaga M, Rosen TA, Levine JD, Julius D. The capsaicin receptor: a heat-activated ion channel in the pain pathway [see comments]. Nature. 1997;389:816-824.

27. Liu Q, et al. Sensory neuron-specific GPCR Mrgprs are itch receptors mediating chloroquine-induced pruritus. Cell. 2009;139(7):1353-1365.
28. Sugimoto Y, Umakoshi K, Nojiri N, Kamei C. Effects of histamine $\mathrm{H} 1$ receptor antagonists on compound 48/80-induced scratching behavior in mice. Eur J Pharmacol. 1998;351(1):1-5.

29. Fleming MS, Ramos D, Han SB, Zhao J, Son YJ, Luo W. The majority of dorsal spinal cord gastrin releasing peptide is synthesized locally whereas neuromedin B is highly expressed in pain- and itch-sensing somatosensory neurons. Mol Pain. 2012;8:52.

30. Martinez A, Zudaire E, Julian M, Moody TW, Cuttitta F. Gastrin-releasing peptide (GRP) induces angiogenesis and the specific GRP blocker 77427 inhibits tumor growth in vitro and in vivo. Oncogene. 2005;24(25):4106-4113.

31. Favata MF, et al. Identification of a novel inhibitor of mitogen-activated protein kinase kinase. J Biol Chem. 1998;273(29):18623-18632.

32. Inagaki $\mathrm{N}$, et al. Inhibition of scratching behavior associated with allergic dermatitis in mice by tacrolimus, but not by dexamethasone. EurJPharmacol. 2006; 546(1-3):189-196.

33. Gober MD, Gaspari AA. Allergic contact dermatitis. Curr Dir Autoimmun. 2008;10:1-26.

34. Saint-Mezard P, et al. Allergic contact dermatitis. Eur J Dermatol. 2004;14(5):284-295.

35. Garcia-Perez A. Occupational dermatitis from DNFB with cross sensitivity to DNCB. Contact Dermatitis. 1978;4(3):125-127.

36. Perez A, Narayan S, Sansom J. Occupational contact dermatitis from 2,4-dinitrofluorobenzene. Contact Dermatitis. 2004;51(5-6):314.

37. Saint-Mezard P, et al. Afferent and efferent phases of allergic contact dermatitis (ACD) can be induced after a single skin contact with haptens: evidence using a mouse model of primary ACD. J Invest Dermatol. 2003;120(4):641-647.

38. Soumelis V, et al. Human epithelial cells trigger dendritic cell mediated allergic inflammation by producing TSLP. Nat Immunol. 2002;3(7):673-680.

39. Yosipovitch G. Dry skin and impairment of barrier function associated with itch - new insights. 
Int J Cosmet Sci. 2004;26(1):1-7.

40. Miyamoto T, Nojima H, Shinkado T, Nakahashi $\mathrm{T}$, Kuraishi Y. Itch-associated response induced by experimental dry skin in mice. Jpn J Pharmacol. 2002; 88(3):285-292.

41. Akiyama T, Carstens MI, Carstens E. Enhanced scratching evoked by PAR-2 agonist and 5-HT but not histamine in a mouse model of chronic dry skin itch. Pain. 2010;151(2):378-383

42. Ji RR, Baba H, Brenner GJ, Woolf CJ. Nociceptive-specific activation of ERK in spinal neurons contributes to pain hypersensitivity. Nat Neurosci. 1999;2(12):1114-1119.

43. Tominaga M, Ogawa H, Takamori K. Histological characterization of cutaneous nerve fibers containing gastrin-releasing peptide in NC/Nga mice: an atopic dermatitis model. J Invest Dermatol. 2009; 129(12):2901-2905.

44. Panula P, Yang HY, Costa E. Neuronal location of the bombesin-like immunoreactivity in the central nervous system of the rat. Regul Pept. 1982; 4(5):275-283.

45. Li MZ, et al. Molecular mapping of developing dorsal horn-enriched genes by microarray and dorsal/ventral subtractive screening. Dev Biol. 2006; 292(2):555-564.

46. Wada E, Way J, Lebacq-Verheyden AM, Battey JF. Neuromedin B and gastrin-releasing peptide mRNAs are differentially distributed in the rat nervous system. J Neurosci. 1990;10(9):2917-2930.
47. Mishra SK, Hoon MA. The cells and circuitry for itch responses in mice. Science. 2013; 340(6135):968-971.

48. Zhang FX, et al. Inhibition of inflammatory pain by activating B-type natriuretic peptide signal pathway in nociceptive sensory neurons. J Neurosci. 2010; 30(32):10927-10938

49. Zhuang ZY, Xu H, Clapham DE, Ji RR. Phosphatidylinositol 3-kinase activates ERK in primary sensory neurons and mediates inflammatory heat hyperalgesia through TRPV1 sensitization. J Neurosci. 2004; 24(38):8300-8309.

50. Obata K, et al. Differential activation of extracellular signal-regulated protein kinase in primary afferent neurons regulates brain-derived neurotrophic factor expression after peripheral inflammation and nerve injury. J Neurosci. 2003; 23(10):4117-4126.

51. Wellbrock C, Karasarides M, Marais R. The RAF proteins take centre stage. Nat Rev Mol Cell Biol. 2004; 5(11):875-885

52. Marinissen MJ, Gutkind JS. G-protein-coupled receptors and signaling networks: emerging paradigms. Trends Pharmacol Sci. 2001;22(7):368-376.

53. Nattkemper LA, et al. Over-expression of the gastrin-releasing peptide in cutaneous nerve fibers and its receptor in spinal cord in primates with chronic itch [published online ahead of print April 4, 2013]. J Invest Dermatol. doi: $10.1038 /$ jid.2013.166
54. Kagami S, et al. Serum gastrin-releasing peptide levels correlate with pruritus in patients with atopic dermatitis. J Invest Dermatol. 2013;133(6):1673-1675.

55. Roberts PJ, Der CJ. Targeting the Raf-MEK-ERK mitogen-activated protein kinase cascade for the treatment of cancer. Oncogene. 2007; 26(22):3291-3310.

56. Hampton LL, et al. Loss of bombesin-induced feeding suppression in gastrin-releasing peptide receptor-deficient mice. Proc Natl Acad Sci U S A. 1998; 95(6):3188-3192.

57. Gong S, et al. A gene expression atlas of the central nervous system based on bacterial artificial chromosomes. Nature. 2003;425(6961):917-925

58. Yamashita H, Makino T, Mizukami H, Nose M. Pharmacological characterization of a chronic pruritus model induced by multiple application of 2,4,6-trinitrochlorobenzene in NC mice. Eur J Pharmacol. 2007; 563(1-3):233-239.

59. Zhao $Z$ Q et al. $L m x 1 b$ is required for maintenance of central serotonergic neurons and mice lacking central serotonergic system exhibit normal locomotor activity. J Neurosci. 2006;26(49):12781-12788.

60. Evans MS, Cheng X, Jeffry JA, Disney KE, Premkumar LS. Sumatriptan inhibits TRPV1 channels in trigeminal neurons. Headache. 2012;52(5):773-784.

61. Demehri S, Morimoto M, Holtzman MJ, Kopan R. Skin-derived TSLP triggers progression from epidermal-barrier defects to asthma. PLoS Biol. 2009; 7(5):e1000067. 